\title{
Efficient calculations of a large number of highly excited states for multiconfigurational wavefunctions
}

\author{
Mickael G. Delcey, Lasse Kragh Sørensen, Morgane Vacher, \\ Rafael Carvalho Couto and Marcus Lundberg*
}

February 27, 2019

\begin{abstract}
Electronically excited states play important roles in many chemical reactions and spectroscopic techniques. In quantum chemistry, a common technique to solve excited states is the multi-root Davidson algorithm, but it is not designed for processes like X-ray spectroscopy that involves hundreds of highly-excited states. We show how the use of a restricted active space wavefunction together with a projection operator to remove low-lying electronic states offers an efficient way to reach single and doublecore hole states. Additionally, several improvements to the stability and efficiency of the configuration interaction (CI) algorithm for a large number of states are suggested. When applied to a series of transition metal complexes the new CI algorithm does not only resolve divergence issues but also leads to typical reduction in computational time by $70 \%$, with the largest savings for small molecules and large active spaces. Together, the projection operator and the improved CI algorithm now makes it possible to simulate a wide range of single- and two-photon spectroscopies.
\end{abstract}

Keywords: Configuration interaction, Excited states, X-ray spectroscopy, multiconfigurational wavefunction, computational cost

*Department of Chemistry - Ångström Laboratory, Uppsala University, S-75105 Uppsala, Sweden 


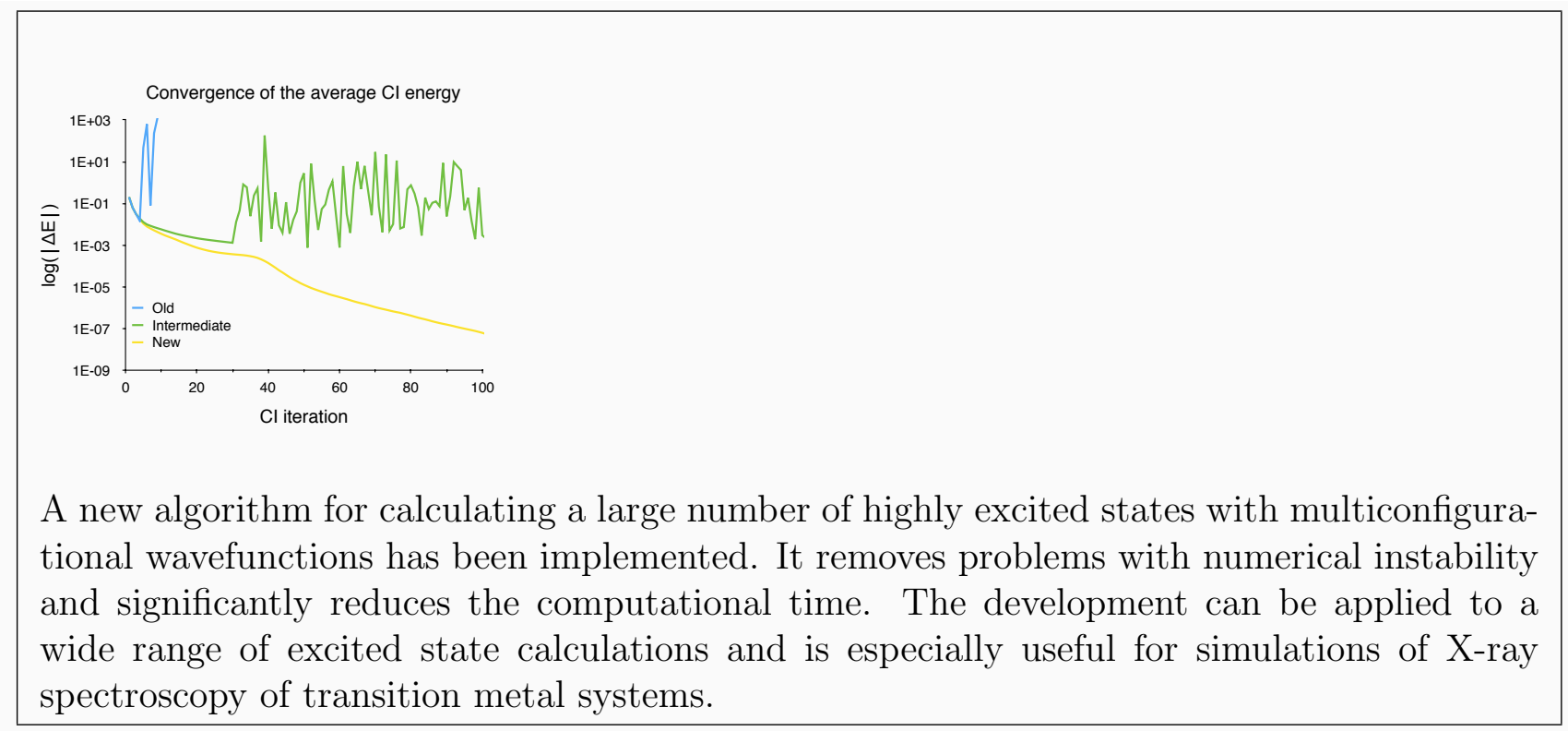




\section{INTRODUCTION}

Theoretical chemistry is often used to outline reaction pathways and to interpret experimental spectra, which requires calculations of both ground and excited state properties. The latter can be done either through a response theory calculation on the ground state or by explicitly calculating the relevant excited states. This second approach is particularly useful for many high-level wavefunction methods as all relevant response equations may not yet be available.

Most standard techniques to compute electronically excited states are designed to target the few lowest energy states. However, many experimental processes target highly excited states, a larger number of states, or a combination of both. A prominent example is X-ray spectroscopy where the sample interacts with high-energy photons, leading to the creation of core-hole states. The energy required to excite core electrons are element specific, which makes it possible to target specific elements in complex systems. The signal is also sensitive to the local structure giving information about spin, electronic structure and bonding. ${ }^{1}$

In recent years there has been an increased focus on the development and use of ab-initio methods to get accurate and unbiased simulations of X-ray processes, as recently reviewed by Norman and Dreuw. ${ }^{2}$ As the physics is essentially the same, most of ab-initio methods for X-ray simulations evolved from standard methods for UV-visible spectrum simulation such as configuration interaction (CI), algebraic diagram construction (ADC) ${ }^{3,4}$, coupled-cluster $(\mathrm{CC})^{5-7}$, time-dependent (TD) density-functional theory (DFT) ${ }^{8,9}$ and multiconfigurational (MC) self-consistent field (SCF) ${ }^{10,11}$ approaches. However, these methods have to be modified from their standard formulation in order to efficiently describe X-ray processes. For

response-theory approaches like the complex polarization propagator (CPP) approach ${ }^{12}$ the $^{-}$ total absorption can be directly computed for a given photon energy, while for real-time methods like real-time $\mathrm{TD}^{-\mathrm{DFT}^{8}}$ the entire spectrum can be computed at once.

For explicit calculations of excited states, several ideas have been suggested to be able to directly target the desired energy range, such as energy-screening algorithms ${ }^{13}$, shifted Davidson algorithms, where states whose energy is close to the shift energy are found, ${ }^{14}$ or the Core-Valence Separation $(\mathrm{CVS})^{15}$. The latter is one of the older and conceptually 
simpler ideas, as it relies on neglecting the mixing between the valence and the core-excited states. This is motivated by the small couplings between those states in the Hamiltonian due to the large energy differences involved. One advantage is that only the core excited states appear in the calculation. Another advantage is the decoupling of the core-hole states from the continuum. As the core-hole states are much higher in energy than the ionization potential, they are embedded in a continuum of high-energy valence states. ${ }^{16}$ To properly describe a core-hole states, one should thus in principle compute the coupling between these states and the continuum. However, with Hermitian mechanics and a finite atom-centered basis set, it is challenging to describe the continuum and thus the coupling. Solutions using complex-valued approaches exist but are associated with increased cost. ${ }^{17}$ Simply neglecting the continuum coupling altogether using CVS gives good results and is better than using a poor continuum description. ${ }^{18}$

One important family of methods that has seen significant use to simulate X-ray spectroscopy is multiconfigurational methods. ${ }^{10,11,19}$ As these methods can also treat systems with near-degenerate valence states, they have been used extensively to model X-ray spectra of transition-metal and rare-earth systems. ${ }^{20-29}$ A majority of these calculations have taken advantage of the flexible structure of the restricted active space self-consistent field (RASSCF) wavefunction, for which dynamical correlation can be added through second-order perturbation (PT2). ${ }^{30-32}$ Transition intensities are calculated using the RAS state-interaction (SI) approach, which also includes spin-orbit coupling. ${ }^{33,34}$ Light-matter interactions can either be calculated using the electric dipole approximation, the full second-order expansion of the wavevector, ${ }^{35-37}$ or the exact semi-classical light-matter interaction. ${ }^{38-40}$ Calculations with RAS wavefunctions have already been used to study covalency in metal-ligand bonding, ${ }^{41}$ identify transient reaction intermediates in femtosecond X-ray spectroscopy, ${ }^{27}$, and to assign low-lying electronic states through their spectral fingerprints. ${ }^{42}$

In most processes, only a single core excitation is required which makes the RAS, where the number of excitations can be restricted, efficient to use compared to complete active space (CAS). However, to reach the core excited states in a standard RAS calculation would typically require the calculation of all valence states, which for a medium-sized active space could be hundreds of thousands of states. Another complication is the number of final states 
required to describe some $\mathrm{X}$-ray processes. An example is the metal L-edge $(2 \mathrm{p} \rightarrow 3 \mathrm{~d}$ excitations) X-ray absorption spectroscopy (XAS) that directly probes the $3 \mathrm{~d}$ orbitals involved in metal-ligand bonding and redox reactivity. ${ }^{1,43-45}$ The full spectrum spans over more than $20 \mathrm{eV}$ and considering the high density of states of transition metals, this translates to a large number of states. Taking ferricyanide $\left(\left[\mathrm{Fe}(\mathrm{CN})_{6}\right]^{4-}\right)$ as an example, the L-edge XAS spectrum shows an intense peak that is a signature of significant $\pi$-backbonding, see Figure 1. This peak is not included in the spectrum when using up to 480 final states per spin multiplicity, and the shape of the peak is not converged until more than 960 states have been included. For this symmetric system, not all states are in the same irreducible representation, but still hundreds of states must be calculated at the same time.

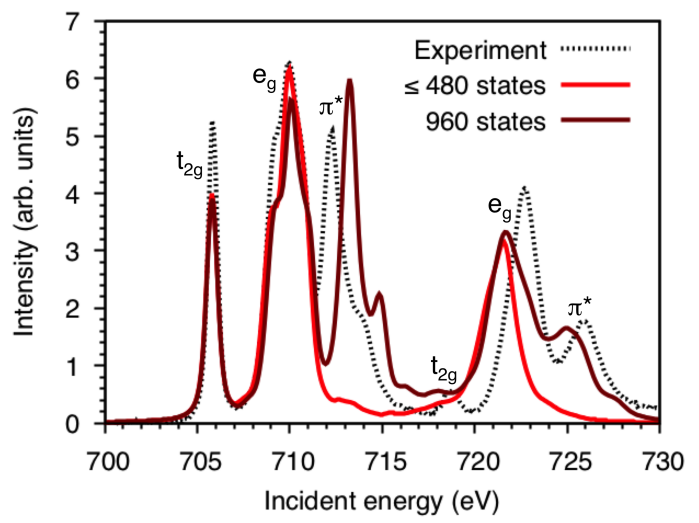

Figure 1: Effect of changing the number of final states per spin multiplicity for restricted active space simulations of ferricyanide $\left(\left[\mathrm{Fe}(\mathrm{CN})_{6}\right]^{4-}\right) \cdot{ }^{46}$ A large number of states is required to predict the $\pi^{*}$ peak in the experimental spectrum that is a signature of $\pi$-backbonding. ${ }^{47}$

However, while it is sometimes possible to use RAS to simulate core-hole spectra in its standard form as shown in Figure 1, the Davidson algorithm is not designed to handle these situations. Here we finally address these issues and implement several modifications designed to improve the efficiency of multiconfigurational methods, specifically the OpenMolcas RASSCF implementation, when dealing with a large number of core-hole states. ${ }^{48}$ We illustrate these improvements for combinations of small and large molecules, with small and large active spaces, using systems ranging from carbon monoxide to an iron complex used as photosensitizer in artificial photosynthesis. 


\section{THEORY}

Here we will explain the various modifications of the CI algorithm we propose to efficiently compute a large number of highly excited states. We start by reviewing the general theory behind MCSCF methods, before discussing how to specifically target highly excited states. Finally, we will show how to improve the efficiency of the algorithm when dealing with a large number of states.

\section{General MCSCF theory}

For a given state $\mathrm{j}$, the wavefunction $\left|\Psi_{j}\right\rangle$ can be written as a linear combination of configuration state functions (CSF) $\left|\Phi_{k}\right\rangle$.

$$
\left|\Psi_{j}\right\rangle=\sum_{k} C_{j, k}\left|\Phi_{k}\right\rangle
$$

where the CSF $\left|\Phi_{k}\right\rangle$ are spin-adapted linear combinations of Slater determinants. In MCSCF theory, a limited set of configurations is included, and the configuration interaction (CI) coefficients $C_{j, k}$ are optimised together with the orbital shapes.

Simulating an optical spectrum typically requires the calculation of the transition energies and intensities between an initial and all relevant final states for the energy range under consideration. One of the most common way to do this in MCSCF theory is to do a state-averaged calculation computing all the states of a given spin and spatial symmetry simultaneously. This means that all excited states use the same orbitals but their own CI coefficients. For small CI spaces, typically a few hundred CSF, the CI coefficient vectors and energies for all states can readily be obtained by diagonalising the Hamiltonian. However, for larger CI spaces, the cost of storing and diagonalising the large Hamiltonian matrix is prohibitive and instead, the lowest energy states are typically found using the iterative Davidson algorithm $^{49}$ or its variant the Davidson-Olsen algorithm ${ }^{50}$. A detailed description of the Davidson algorithm and strategies for addressing core-hole states are given in reference $^{14}$. Here only a brief description relating to our implementation is given.

The Davidson-Olsen algorithm is simply the Quasi-Newton method applied to the CI problem. As for any Newton algorithm, the Hessian inverse is required, and in the case of 
the CI optimisation, the electronic Hessian - the second derivative of the energy with respect to the CI coefficients - is related to the Hamiltonian $\mathbf{H}$. The Davidson or Davidson-Olsen algorithm thus requires an approximate Hamiltonian, $\mathbf{H}_{0}$, typically a diagonal approximation, to easily calculate the inverse needed at each iteration. Following the notations in ref. ${ }^{51}$, the CI coefficients vectors are denoted with a capital $\mathbf{C}$ while the Davidson corrections use the small case $\mathbf{c}$. At an iteration $i$, the Davidson correction for state $j, \mathbf{c}_{j}^{(i)}$, can be computed using

$$
\mathbf{c}_{j}^{(i)}=-\left(\mathbf{H}_{0}-E_{j}^{(i)} \mathbf{1}\right)^{-1}\left(\mathbf{H}-E_{j}^{(i)} \mathbf{1}\right) \mathbf{C}_{j}^{(i)}
$$

with $\mathbf{C}_{j}^{(i)}$ the CI vector for state $j$ at the current iteration and $E_{j}^{(i)}$ its energy. The DavidsonOlsen equation is somewhat more complex but has the advantage that the correction vector is always orthogonal to the initial CI vector, even if $\mathbf{H}_{0}$ approaches $\mathbf{H}$, at which point the simple Davidson would completely fail.

While equation 2 could, in principle, be used directly to improve on $\mathbf{C}_{j}^{(i)}$, for added flexibility the next CI vector $\mathbf{C}_{j}^{(i+1)}$ is instead typically found by diagonalising a small Hamiltonian in the basis of all CI vectors $\mathbf{C}_{j}^{(i)}$ at the previous iteration and the corresponding correction vectors $\mathbf{c}_{j}^{(i)}$. To improve convergence even further, the correction vectors of a few previous iterations are often added to the small Hamiltonian. This diagonalisation is in most cases inexpensive and the vast majority of the computational cost of the algorithm comes from the computation of the so-called sigma vector:

$$
\boldsymbol{\sigma}_{j}^{(i)}=\mathbf{H c}_{j}^{(i)}
$$

which has to be done for each state at each iteration.

In a first order MCSCF code, once the CI coefficients have been found in this iterative procedure (microiterations), one step of orbital optimization (macroiteration) is performed based on the average density matrix from all the states. Then the CI optimisation is restarted based on these new orbitals. This iterative process continues until simultaneous convergence of the CI and orbital optimisations. For efficiency purposes, in OpenMolcas, the CI convergence thresholds are rather loose in the beginning and only tighten as the orbitals approach the converged solution. For example, by default, the CI convergence energy threshold is 
chosen to be one thousandth of the energy change in the past macroiteration. On the other hand, the final convergence thresholds are rather tight by default, with for example $1.0 \mathrm{~d}^{-8}$ ha as energy threshold for the orbital optimisation (implying $1.0 \mathrm{~d}^{-11}$ ha for the CI step). Additionally, the maximum number of allowed CI steps is capped to 12 in the beginning and the cap is slowly lifted by 12 at every macroiteration with a maximum of 500 .

\section{Targeting highly excited states}

What separates different MCSCF methods is the way to choose the configurations. In CASSCF all possible excitations within a selected set of active orbitals are included. In the RASSCF in OpenMolcas, the active space is divided into subspaces: RAS1 with an upper limit on the number of holes, RAS2 where all excitations are allowed, and RAS3 with an upper limit on the number of electrons. For descriptions of X-ray spectra, the core orbitals must be included in the active space and in RAS it is natural to place the core orbitals in the RAS1 space allowing for a maximum of one hole. ${ }^{21-23}$ The valence orbitals can then be distributed among RAS2 and RAS3 spaces to get a manageable number of valence configurations. ${ }^{21,25,41}$ For processes involving holes in more than one set of core orbitals, like core-to-core emission or resonant inelastic X-ray scattering, two different RAS spaces can be used instead to independently control the occupations in the different sets. ${ }^{52}$

Thanks to its inherent flexibility, RAS has been applied to core-hole excited states without specific tailoring. With core orbitals in RAS1, the most straightforward way to generate core-excited states is to calculate all valence excited states (where RAS1 is fully occupied), followed by the core-excited states. For small valence active spaces, e.g., five electrons in the five metal 3d-dominated orbitals in a first-row transition metal complex, only a few hundreds of valence states need to be calculated. ${ }^{23,53}$ Although the computational cost is approximately linear with the number of states, hundreds of core-excited states are anyway required so the increase in cost is not that dramatic. However, for a standard RAS2 space with 10 electrons in 10 orbitals, hundreds of thousands of states would be required, which is prohibitive.

Presence of symmetry can significantly help to reach core-excited states. A clear example are the $\mathrm{p} \rightarrow \mathrm{d}$ transitions in centrosymmetric transition metal complexes. Here the $\mathrm{p}$ orbitals 
are ungerade while all relevant valence orbitals are gerade, in which case the lowest ungerade state must have a core hole. ${ }^{21,22,25}$

A number of calculations have also been performed by putting the core orbitals in RAS3 allowing $N-1$ electrons in this space with $N$ the number corresponding to full orbital occupation. ${ }^{46,54}$ This has two main drawbacks. First, all configurations with $N-2, N-3 \ldots$ 0 electrons in the core orbitals were also computed, increasing the computational cost even though those have insignificant contributions to the final wavefunctions. ${ }^{46}$ Second, transition intensities and/or spin-orbit coupling for the RASSCF wavefunctions are calculated using RASSI $^{33,34}$, which in its current implementation requires the CI expansions in the RASSCF calculation for all states of the same symmetry to be identical. This is not the case here as configurations with $N$ electrons in RAS3 are present in the ground and excited valence states but excluded from the core-excited states.

As an efficient and generally applicable alternative to these techniques we have implemented a projection technique, based on the CVS approximation. ${ }^{15}$ With core orbitals in RAS1, the initial states are calculated in the usual RASSCF fashion though configurations with holes in RAS1 are included. The core-excited states are then found by running a separate RASSCF calculation with the same active space definitions but where we formally apply a core-hole (ch) projection operator $P_{c h}$

$$
P_{c h} \Psi=\Psi_{c h}
$$

on the CI wavefunctions, which for single-core-hole excited states removes all configurations in $\Psi$ that are fully occupied in RAS1. For conveniency, we will refer to this as the highly excited state (HEXS) procedure. If the target is double-core-hole excited states both configurations fully occupied in RAS1 and configurations with a single hole in RAS1 are removed, hereafter referred to as the DEXS procedure. The projection operator $P_{c h}$ ensures that the lowest state in $\Psi_{c h}$ is the first single or double core-excited state since all configurations included all have at least one or two core holes, respectively. It is worth noting that the exclusion of the valence excited states not only reduces the number of states needed to be calculated but also ensures that the undesired states do not negatively impact the quality of the orbitals for the core-excited states. 
In Openmolcas ${ }^{48}$ the projection operator $P_{c h}$ is particularly simple thanks to the generalised active space (GAS) structure of the CI module Lucia. ${ }^{30,55}$ Indeed, in GAS, the CI expansion is separated in blocks corresponding to a specific number of electrons in each active space. The projection operator simply corresponds to finding the blocks in the CI expansion where RAS1 is either fully occupied or have a single hole, for single- and doublecore-excited states respectively, and setting these to zero in the sigma vector step in Eq. 3 and in the optimisation step. Since the parts zeroed out are excluded in the sigma vector step and optimisation, the calculation of a core-excited state is cheaper than the calculation of a ground or valence-excited state.

In this way the CI expansion for the initial and final states are the same, albeit with many trivially zero CI-coefficients in the core-excited states. This simplifies the calculations of the transition intensities in the state-interaction calculations. Formally, by performing the calculation of the initial and final separately, with separate orbital optimisation, the initial and final states are not strictly orthogonal, but transition intensities can still be efficiently calculated by taking advantage of the biorthogonalisation in RASSI. ${ }^{56}$

It is useful to note the relation between the present approach and the CVS approximation. A key feature of the latter is that it removes the coupling to the continuum states, which in standard approaches are only approximately modelled using excitations into pseudo-continuum, probably very diffuse, orbitals. ${ }^{16,57}$ The RASSCF approach only include excitations into active orbitals, which are typically low-lying localised orbitals. This excludes the pseudo-continuum in virtually all cases, even if core and valence states would remain coupled. The main benefit of the HEXS approximation compared to the standard RAS approach is thus only that valence excited states do not have to be calculated.

\section{Improving the $\mathrm{Cl}$ convergence}

Even if the algorithm can be directed to directly target the core-hole states, the number of states necessary to fully describe a spectrum can be very high, typically on the order of several hundreds, see Figure $1 .{ }^{46}$ As mentioned above, this can create difficulties for standard Davidson algorithms. When computing hundreds of states, storing several hundreds of CI or correction vectors for a few iterations can put high constraints on the memory. These vectors 
also tend to have significant linear dependency, which can lead to numerical instability during the orthonormalisation while forming the small Hamiltonian and in the worst cases result in divergence of the Davidson algorithm. Additionally, high-lying excited states have poor convergence behaviour leading to a very high number of iterations which can become very expensive. This is in large part because highly excited states are less likely to be dominated by one or a few determinants, and the diagonal approximation $\mathbf{H}_{0}$ in eq. 2 is therefore inaccurate.

The most straightforward way to reduce problems with linear dependency is to reduce the number of variables. The original version of the OpenMolcas CI algorithm, hereafter referred to as Version 1, used five past iterations for which the corrections vectors were used in the diagonalisation. For a calculation with 120 states, this corresponds to 600 correction vectors on top of the 120 CI vectors, each with a length corresponding to the full CI expansion. Reducing this number to 2 past iterations, here called Version 2, gives only a total of 360 vectors and is a straightforward way to reduce the risk of divergence, but it also reduces the flexibility in the diagonalisation and can thus be expected to slow down the convergence. To find a CI algorithm that is both quick and stable requires more significant changes.

The Davidson-Olsen iterations were made more stable by modifying the orthonormalisation algorithm from the classical to the modified Gram-Schmidt, including the possibility to drop linearly-dependent vectors. Additionally, the sigma vectors corresponding to the updated CI vectors are usually simply computed at each iteration as linear combinations of the sigma vectors for the previous CI and correction vectors. As the number of CI iterations sometimes gets very high, the errors accumulate over time. To remedy this, the CI vectors are renormalised after the diagonalisation, and every 24 CI iterations, the sigma vectors corresponding to the current CI vectors are recomputed from scratch from the CI coefficients. Those extra steps add some computational overhead but should significantly reduce the risk of divergence. Further, instead of storing a fixed number of old correction vectors for each state, a global cap can be applied to the number of vectors. This makes the algorithm less sensitive to the number of states. The default value was chosen to be 400 total vectors, though ensuring that at least 2 and at most 5 previous iterations are stored. As soon as the limit is reached, old vectors are simply replaced by newer ones. The version containing all 
these changes is called Version 3.

Versions 1-3 treat all states equally, but the convergence behaviour of low and high-energy roots are often very different, with the former converging much faster than the latter. An improvement with the potential of significant time savings is to stop computing correction vectors for the low-lying states when they satisfy the convergence criteria, even if higher-lying states are still not converged. As the lowest roots usually converge within a few iterations, this should allow for a drastic reduction of the number of sigma vectors to compute during the majority of the Davidson-Olsen iterations. This can lead to significant cost reductions even if the total number of CI iterations does not decrease. By adding this change to Version 3, our final algorithm is obtained which will be referred to as Version 4. A summary of the main features of those four version is shown in table 1.

\begin{tabular}{c|l|l|l|l}
\hline Version & Gram-Schmidt & $\begin{array}{l}\text { Renormalise } \\
\text { CI vectors }\end{array}$ & $\begin{array}{l}\text { Treatment of } \\
\text { converged states }\end{array}$ & max number of states \\
\hline 1 & Conventional & No & Compute & 6 x nstates \\
2 & Conventional & No & Compute & 3 x nstates \\
3 & Modified & Yes & Compute & $\min (6$ x nstates, 400) \\
4 & Modified & Yes & Skip & $\min (6$ x nstates, 400) \\
\hline
\end{tabular}

Table 1: Summary of the main features of the four versions of the CI code.

\section{COMPUTATIONAL DETAILS}

To highlight the capabilities of our implementation of core-valence separation to compute highly excited states, four small molecules were selected: methane, carbon monoxide, carbon dioxide and carbon disulfide. To illustrate the increased efficiency of the new Davidson implementation, four transition metal complexes, $\mathrm{Mn}_{2}^{+}, \mathrm{MnO}_{2}^{+},\left[\mathrm{Fe}(\mathrm{P})(\mathrm{ImH})_{2}\right]^{+}(\mathrm{P}=$ porphyrin, $\mathrm{ImH}=$ imidazole $)$, and $\left[\mathrm{Fe}(\mathrm{bmip})_{2}\right]^{2+}[\mathrm{bmip}=2,6$-bis(3-methyl-imidazole-1-ylidine)-pyridine $]$ (see Figure 2), were computed using the different versions of the CI algorithm described in the previous section. These four complexes represent combinations of small and large molecules with small and large active spaces and showcase different situations with respect 
to the relative cost of the CI and the orbital optimisations.

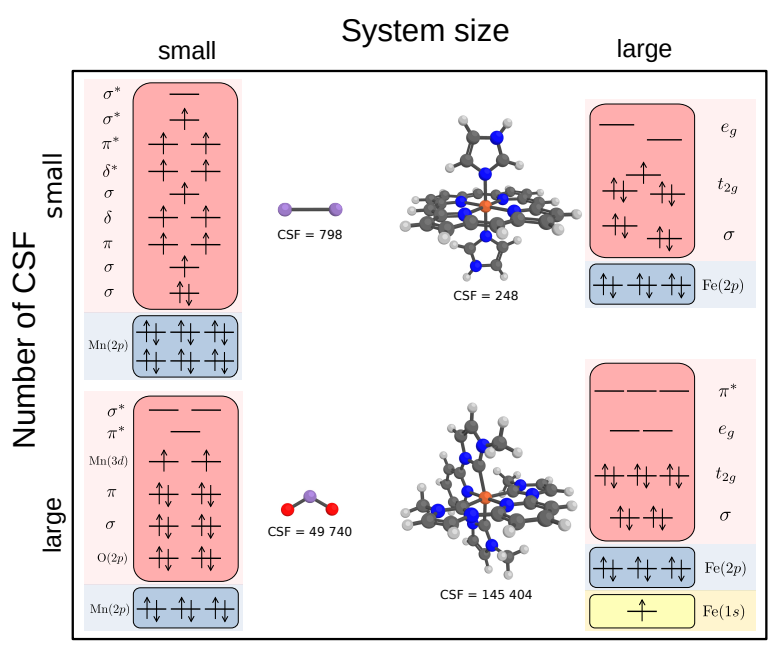

Figure 2: The four transition-metal systems used to test the CI algorithm. The active spaces and initial state configurations are displayed on the side. The colored boxes indicate the ras spaces, with blue, red and yellow representing RAS1, RAS2, and RAS3 respectively.

In all RASSCF calculations the relevant valence orbitals were placed in RAS2. Unless stated otherwise, active core orbitals were placed in RAS1 allowing one or two excitations depending on whether the target is single or double core-hole states. In all cases, the active core orbitals were frozen in the RASSCF optimization in order to avoid orbital rotation. Symmetry is used whenever possible and RASSCF calculations are run independently for each irreducible representation. Douglas-Kroll-Hess scalar relativistic effects are included in combination with the ANO-RCC basis set and Cholesky-based resolution of the identity auxiliary basis. ${ }^{58-62}$ Triple-zeta contraction was used for all systems except for $[\mathrm{Fe}(\mathrm{P})(\mathrm{ImH})]^{+}$ and $\left[\mathrm{Fe}(\mathrm{bmip})_{2}\right]^{2+}$ which used double-zeta contraction. Spin-orbit coupling was included using the RASSI-SO method. ${ }^{34}$ All calculations are performed with the OpenMolcas program package.

For the small organic molecules, the carbon K-edge (1s excitations) was modelled. The RAS1 spaces consisted of the carbon 1s orbital, while the RAS2 spaces consisted of 8 electrons distributed in 9 orbitals $(8,9)$ for $\mathrm{CH}_{4},(10,14)$ for $\mathrm{CO},(12,12)$ for $\mathrm{CO}_{2}$ and $(12,13)$ for $\mathrm{CS}_{2}$. As the goal was to find the edge position, only one initial and one final states were computed. To get better agreement with experimental data, RASSCF results are corrected using multi- 
state second-order perturbation theory $\left(\right.$ RASPT2) ${ }^{32}$.

For the transition-metal complexes, all processes involve states with metal $2 \mathrm{p}$ holes, either L-edge XAS $(2 \mathrm{p} \rightarrow 3 \mathrm{~d})$ or $\mathrm{K} \alpha$ emission $(2 \mathrm{p} \rightarrow 1 \mathrm{~s})$. Here spin-orbit coupling plays an important role and $2 \mathrm{p}$ core-hole states are, unless otherwise stated, computed both in the initial spin state $(\mathrm{S})$ and with $S=S \pm 1 . \mathrm{Mn}_{2}^{+}$and $\mathrm{MnO}_{2}^{+}$are both small with 118 and 119 basis functions respectively. $\mathrm{Mn}_{2}^{+}$has a total of 19 orbitals in the active space, 6 core and 13 valence (see Figure 2), but a very high ground-state spin multiplicity $(S=5)$, thus the number of configurations is still small (798 CSF). According to the spin-orbit rules, states with $S=5 \pm 1$ should also be calculated, but in order to serve as an example with a small number of configurations, which is not true for the $S=4$ states, only $S=5$ states are included. Accounting for symmetry, there are 6 independent final-state RASSCF calculations, each with 120 states, equivalent to the calculations of $\left[\mathrm{Fe}(\mathrm{CN})_{6}\right]^{4-}$ in Figure $1 . \mathrm{MnO}_{2}^{+}$has triplet ground state multiplicity and 11 valence orbitals in the active space, which gives tens of thousands of configurations for the ground state. Here singlet, triplet and quintet final states are needed, additionally split in the 4 different irreducible representations of $C_{2 v}$, for a total of 12 independent RASSCF calculations with 120 states each.

The third case is the low-spin $(\mathrm{S}=1 / 2)$ iron porphyrin $\left[\mathrm{Fe}(\mathrm{P})(\mathrm{ImH})_{2}\right]^{+}$. This molecule is significantly larger with a total of 610 basis functions even with a double-zeta contraction. The simulated L-edge spectra has been published previously. ${ }^{42}$ Those results were obtained using 10 orbitals in the valence active space, but here a smaller active space of seven valence orbitals is used to reduce the number of CSF, see Figure 2. Both doublet and quartet final states are computed in the two ungerade irreducible representations of the $C_{2 h}$ point group for a total of 4 RASSCF calculations with 60 states each. The calculated spectrum is generated using a Lorentzian broadening with a full-width-at-half-maximum (FWHM) of 0.4 and $0.8 \mathrm{eV}$ for the $L_{3}$ and $L_{2}$ edges respectively, and convoluted with an experimental Gaussian broadening of $0.4 \mathrm{eV}$.

The fourth and final transition-metal complex is $\left[\mathrm{Fe}(\mathrm{bmip})_{2}\right]^{2+}$, which is also a relatively large molecule with 668 basis functions at the double-zeta level. To model K $\alpha$ emission, both $2 \mathrm{p}$ and 1 s orbitals need to be included, and these are put in RAS1 and RAS3 respectively. As RAS3 does not restrict the number of holes and thus leads to more configurations, ideally 
the smaller set of core orbitals is put there while the larger set should be in RAS1. The valence active space consists of 10 orbitals, which for the the initial 1s-excited state $(\mathrm{S}=1 / 2)$ has more than hundred thousand CSF, see Figure 2. The doublet final states with a 2p core-hole states are spread over 3 different irreducible representations of the $D_{2}$ point group, with respectively 70,70 and 90 states.

The computational times are given for the RASSCF module in a serial execution of OpenMolcas on Intel Xeon Gold 6130 processors (base frequency of $2.10 \mathrm{GHz}$ ). All calculations involve several independent RASSCF calculations with different symmetry and/or spin multiplicities, and the time and CI iterations shown are the total over those calculations.

\section{RESULTS AND DISCUSSION}

\section{Highly excited states}

The ability to select CSF in the RASSCF procedure allows for direct calculations of single and double vacancies of deep orbitals, while at the same time reducing the computational cost. This is illustrated for five different carbon 1s core-excitation: single core-excited $\left(K^{-1} V^{1}\right)$, single core-ionised $\left(K^{-1}\right)$, double core-excited $\left(K^{-2} V^{2}\right)$, core-ionised-core-excited $\left(K^{-2} V^{1}\right)$, and double core-ionised $\left(K^{-2}\right)$. Double-core-hole states are significantly less common than single-core-hole ones. However, they lead to an enhancement of the chemical sensitivity and have therefore gained renewed attention with the appearance of new intense X-ray lightsources. ${ }^{63-68}$

Taking the $\mathrm{CO}_{2}$ molecule as an example, the highly excited states (HEXS) procedure for the computation of single core-excited/-ionised states leads to a reduction of around $26 \%$ in the number of CSF, see Table 2. These forty thousand CSF correspond to valence excited states that would have to be computed before reaching the first states with 1s core holes if they had to be calculated by order of energy. For the calculations of double coreexcited/-ionised, the doubly excited state (DEXS) procedure reduces the number of CSF by an average of $73 \%$, see Tab. 2 .

The chemical sensitivity of the X-ray spectra can be seen from the differences in excitation 
Table 2: Number of CSF, highly excited CSF and the number of excluded CSF (percentage in parentheses) when using HEXS or DEXS approaches. The results are from the $\mathrm{CO}_{2}$ molecule and are considered for the ground state (GS), single core-excited $\left(K^{-1} V^{1}\right)$, single core-ionised $\left(K^{-1}\right)$, double core-excited $\left(K^{-2} V^{2}\right)$, core-ionised-core-excited $\left(K^{-2} V^{1}\right)$, and double core-ionised $\left(K^{-2}\right)$.

\begin{tabular}{lccc}
\hline & CSF & Highly excited CSF & Excluded CSF (\%) \\
\hline GS & 93348 & - & - \\
\hline HEXS & & & $28224(30.71)$ \\
$K^{-1} V^{1}$ & 91908 & 63684 & $42696(26.37)$ \\
$K^{-1}$ & 161919 & 119223 & \\
\hline DEXS & & & $70740(76.97)$ \\
$K^{-2} V^{2}$ & 91908 & 21168 & $118548(73.60)$ \\
$K^{-2} V^{1}$ & 161064 & 42516 & $64404(68.99)$ \\
$K^{-2}$ & 93348 & 28944 & \\
\hline
\end{tabular}

energies between the molecules, see Tab. 3. The single ionisation energies can be separated in two blocks, one containing $\mathrm{CH}_{4}$ and $\mathrm{CS}_{2}$ and another $\mathrm{CO}$ and $\mathrm{CO}_{2}$, with an average difference of $5 \mathrm{eV}$ between them, which reflects the electronegativity of the atoms bound to carbon. The double core-ionised systems show even bigger energy difference between the two blocks of about $14 \mathrm{eV}$. The computed energies agree very well with experimental data in almost all cases, which is rather remarkable considering that the core orbitals are not relaxed and no specific basis set is used to handle contraction of the other orbitals after the core excitation.

\section{Davidson algorithm}

The difficulties to converge the Davidson algorithm becomes more prominent as the number of states increases. In multiconfigurational calculations there are two main considerations that needs to be taken into account when deciding on the number of states. First, the selected states must cover the full energy range of the measured spectrum. Second, the state-average 
Table 3: Carbon K-edge RASPT2 energies obtained using the HEXS and DEXS methods for methane, carbon monoxide, carbon dioxide and carbon disulfide. Are presented the energies (in eV) for the single core-excited $\left(K^{-1} V^{1}\right)$, single core-ionised $\left(K^{-1}\right)$, double coreexcited $\left(K^{-2} V^{2}\right)$, core-ionised-core-excited $\left(K^{-2} V^{1}\right)$, and double core-ionised $\left(K^{-2}\right)$. The experimental values are shown in parentheses.

\begin{tabular}{cccccc}
\hline System & $K^{-1} V^{1}$ & $K^{-1}$ & $K^{-2} V^{2}$ & $K^{-2} V^{1}$ & $K^{-2}$ \\
\hline $\mathrm{CH}_{4}$ & $286.78\left(287.05^{69}\right)$ & $290.63\left(290.76^{69}\right)$ & 633.72 & 639.44 & $650.82\left(651.50^{65}\right)$ \\
$\mathrm{CO}$ & $287.33\left(287.40^{69}\right)$ & $295.90\left(296.05^{69}\right)$ & 638.40 & 645.33 & $665.37\left(667.90^{66}\right)$ \\
$\mathrm{CO}_{2}$ & $290.75\left(290.77^{69}\right)$ & $297.28\left(297.66^{69}\right)$ & 640.42 & $645.27\left(645.60^{67}\right)$ & $665.35\left(666.00^{67}\right)$ \\
$\mathrm{CS}_{2}$ & $286.20\left(286.10^{70}\right)$ & $292.99\left(293.10^{70}\right)$ & 632.27 & $636.53\left(635.00^{68}\right)$ & 651.73 \\
\hline
\end{tabular}

RASSCF calculations must converge to the desired orbitals. Satisfying both these criteria can require the inclusion of a large number of states.

Many X-ray spectra span tens of $\mathrm{eV}$, which coupled with a high density of states of transition-metal complexes, can lead to a very large number of final states. As an illustration, the L-edge XAS spectrum of $[\mathrm{Fe}(\mathrm{P})(\mathrm{ImH})]^{+}$is plotted together with the total number of states below a certain energy, see Figure 3. The number of states displayed in the figure is the number of spin-orbit states, which corresponds to the number of RASSCF states multiplied by their respective spin multiplicity. Even with this small active space, the Ledge XAS spectrum includes more than 700 spin-orbit states.

In the $\left[\mathrm{Fe}(\mathrm{bmip})_{2}\right]^{2+}$ photosensitiser, the absorption of an UV/Vis photon leads to a metal-to-ligand charge transfer (MLCT) excitation and subsequent electron transfer. To be able to describe the MLCT state, the active space requires the inclusion of three empty ligand-dominated orbitals of $\pi^{*}$ character, see Figure 2. However, in the state-average procedure there is a competition between these chemically relevant orbitals and a set of metal 4d-type orbitals that correlate better with the metal 3d, something known as the double-shell effect. ${ }^{72}$ The ligand-dominated orbitals can be made to enter the active space by increasing the number of states until states representing MLCT excitations are included. In the corehole states, this requires a large number of states as the core hole preferentially stabilises metal-centered orbitals over ligand-centered ones. For 2 p core-hole states, between 70 and 


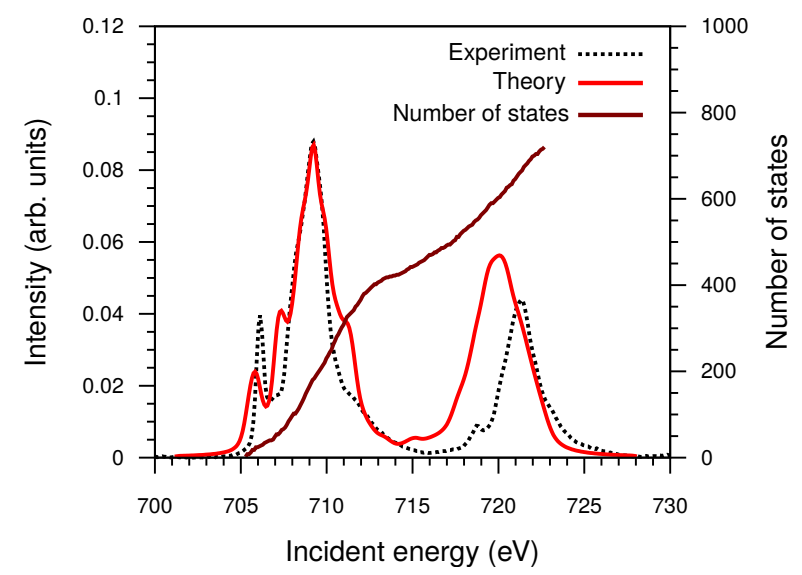

Figure 3: L-edge X-ray absorption spectrum of $[\mathrm{Fe}(\mathrm{P})(\mathrm{ImH})]^{+}$together with the total number of spin-orbit states below a given energy. The experimental spectrum is taken from reference $^{71}$. The RAS simulated spectrum and final-state energies have been shifted by -6.94 $\mathrm{eV}$ in order to compare with the experimental spectrum

90 states per irreducible representation are required to get the $\pi^{*}$ orbitals into the active space.

The comparison between the four versions of the Davidson algorithm described in the theory section for the four transition-metal systems described in the computational details is shown in Figure 4. For most of these systems, the old algorithm (Version 1) diverges in at least one of the RASSCF runs, and thus no timing or iteration count can be shown for this version.

To analyse those results in detail, we focus on the first example, the L-edge XAS spectrum of $\mathrm{Mn}_{2}^{+}$. As this is a small molecule, the CI steps constitute the vast majority of the time spent for the RASSCF optimisation. Thus, any improvement in the Davidson algorithm results in a significant improvement in the total calculation time. Version 1 did not converge, but there is a more than 20-fold reduction in time going from Version 2 to Version 4.

A part of this drastic reduction can be understood by looking at the number of CI iterations, which sees a steep drop going from Version 2 to Version 3 and a more moderate reduction between Versions 3 and 4. In total, the number of CI iterations drops from 16 571 to 1590 . As the number of states for each RASSCF calculation is 120, Version 2 uses 360 vectors while version 3 uses by default 400. This increase in flexibility explains part of 

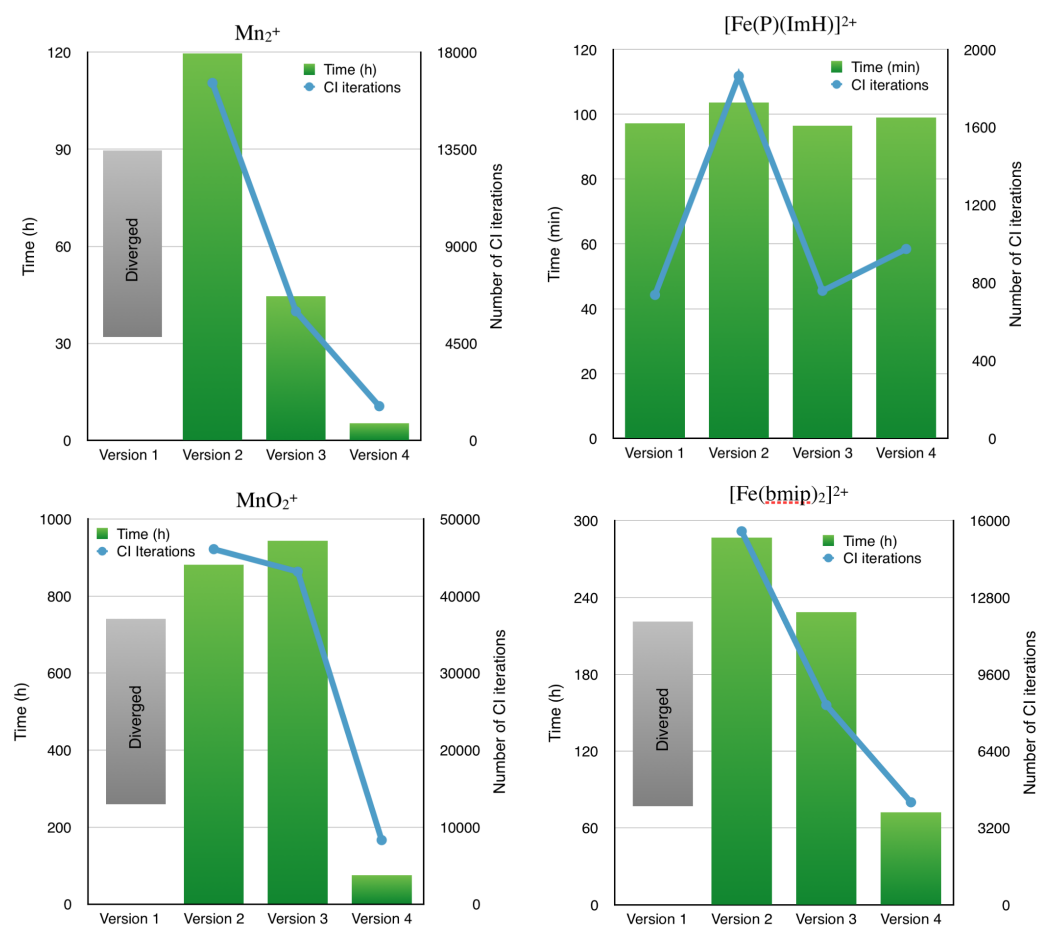

Figure 4: Total RASSCF time and number of CI iterations for the calculation of the Ledge XAS spectra of $\mathrm{Mn}_{2}^{+}, \mathrm{MnO}_{2}^{+}$and $[\mathrm{Fe}(\mathrm{P})(\mathrm{ImH})]^{+}$and the $\mathrm{K} \alpha$ emission spectrum of $\left[\mathrm{Fe}(\mathrm{bmip})_{2}\right]^{2+}$

the faster convergence, but an additional calculation with version 3 using 360 vectors stills shows approximately a factor of 4 improvement over version 2 . It turns out that the drop in the number of iterations is mostly due to the stability improvements in the code, showing their importance even when no divergence is observed. This can be seen by the significantly oscillating convergence of the average energy for one of the RASSCF calculation, see figure 5 .

On the other hand, the reduction in the number of iterations when going from Version 3 to 4 is less straightforward to understand. As these algorithms uses the same number of vectors we could have expected that the latter would save time on the sigma vector step, but not reduce the number of iterations. However, since we only compute correction vectors for the remaining unconverged states, the vectors we store form a good basis for precisely those 

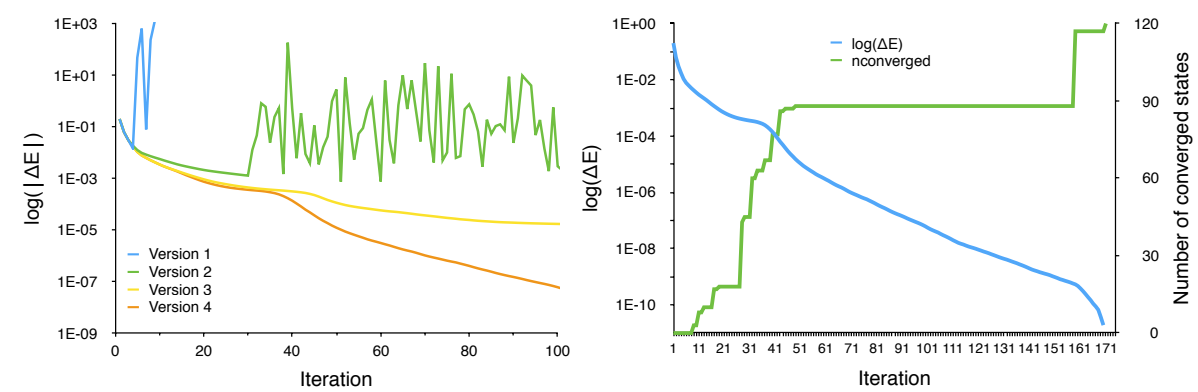

Figure 5: CI convergence for the $\mathrm{a}_{g}$ states of $\mathrm{Mn}_{2}^{+}$. Left panel : averaged energy convergence for each version expressed as the logarithm of the absolute error to the converged energy at each CI iteration. Right panel : Number of converged low energy states for each iteration with Version 4. In both cases, the CI was fully converged from scratch for a given orbital set.

remaining states. Thus while they have the same flexibility in terms of number of vectors, those are more relevant to the remaining states and thus convergence is improved.

The reduction in the number of iterations is clearly a large part of the reduction in total time, but while the number of iterations is divided by more than 3 , the total time is divided by 8 . The iterations are not just fewer but also significantly faster. This confirms that not recomputing the converged states constitutes a significant part of the savings in computational time. In fact, as can be seen in figure 5, most of the iterations are only focusing on the highest 30 to 40 states and their cost is thus about $25 \%$ of the cost of a full iteration.

The conclusions for $\mathrm{Mn}_{2}^{+}$can, to some extent, be applied to the other three cases, with some minor nuances. An important difference is the relative cost of the CI step compared to the orbital step in the RASSCF calculation. For $\mathrm{Mn}_{2}^{+}$and $\mathrm{MnO}_{2}^{+}$, the CI represents the vast majority of the total cost and thus the cost is reduced by more than an order of magnitude going from Version 2 to Version 4. For $[\mathrm{Fe}(\mathrm{P})(\mathrm{ImH})]^{+}$, a large system with a small active space, the CI step is but a small fraction of the total cost and the timing differences between the different versions are very minor. Finally, for $\left[\mathrm{Fe}(\mathrm{bmip})_{2}\right]^{2+}$, both the orbital and the CI steps are computationally intensive, and the cost reduction is a bit more than a factor of 3 , which is still very respectable.

Some other more specific differences can be noted. For $\mathrm{MnO}_{2}^{+}$, the change in the number 
of iterations between Version 2 and 3 is relatively small as the numerical issues are less significant, and thus the cost is actually slightly increased due to the extra costs of the stability checks. On the other hand, for this case, Version 4 represents a significant improvement over Version 3. The reason why the improvement is so large in this case are not clear. Similarly, for $[\mathrm{Fe}(\mathrm{P})(\mathrm{ImH})]^{+}$, numerical issues are nearly non-existent such that Version 1 actually converges and Version 3 represents no improvement over it. The situation is actually worse with Version 4 requiring slightly more iterations. This is likely accidental and not a trend to be expected. Additionally, despite this, the timings for Version 4 remains on par with the original code.

While the results in Figure 4 represent real situations with the number of states chosen to produce converged spectra, the fact that Version 1 diverges for most of them prevents us from getting a good comparison to the timings of the original CI code. To remedy this, we ran additional calculations where the number of state were reduced until Version 1 converges. For $\mathrm{Mn}_{2}$, the number of states had to be decreased to 40 per irreducible representation to obtain convergence, and there, Version 1 took still more than $70 \mathrm{~h}$ to complete with more than 20,000 CI iterations, which is partly due to some remaining numerical instabilities, while Version 4 took about one hour and twenty minutes for a total of 1,751 CI iterations. For $\left[\mathrm{Fe}(\mathrm{bmip})_{2}\right]^{2+}$, Version 1 successfully converged if the number of states was decreased to between 20 and 70 depending on the irreducible representation, and the final time was about $35 \mathrm{~h}$ with 2,405 iterations, while the new code converged in about as many iterations $(2,437)$ but only 20h. This shows that Version 4 is indeed the fastest version even when Version 1 successfully converges due to a smaller number of states.

As increasing the number of kept vectors increases the flexibility in the Davidson iteration, and thus the convergence rate, it is interesting to look specifically at the effect of this number. Figure 6 displays the computational time and number of CI iterations for the calculation of all triplet states of $\mathrm{MnO}_{2}^{+}$in function of the number of kept vectors in Version 4.

A first thing to note is that mainly due to the stability improvements, none of the calculations diverged, even with a large number of kept vectors. Additionally, the number of CI iterations follows a smooth downward trend as the number of vectors increases, reflecting the expected effect of the added flexibility in the diagonalisation. However, the running 


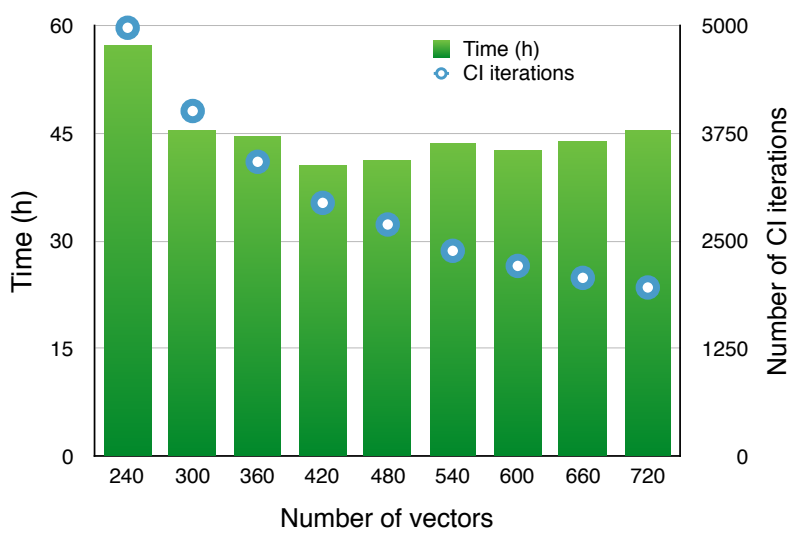

Figure 6: Total RASSCF time and number of CI iterations for the calculation of the 480 triplet excited states of $\mathrm{MnO}_{2}^{+}$as a function of the number of kept vectors.

time does not follow such a clear trend with an optimal value around 420 vectors. This comes from the cost of the creation and diagonalisation of an increasingly large Hamiltonian, including the orthonormalisation, which scales at least quadratically with the number of vectors, negating the benefits of reducing the number of iterations. The optimum seems to be around 400 vectors, which is in line with several other calculations not shown here, and justifies our chosen default value.

Of course, in a real calculation, RASSCF is only a part of the total cost. A typical spectrum calculation with OpenMolcas will include the calculation of the integrals usually with the help of the Cholesky-decomposition-based resolution of the identity ${ }^{62,73}$, as well as the state interaction (RASSI) ${ }^{33}$ calculation to estimate transition intensities and/or to compute the spin-orbit coupling elements. ${ }^{34}$ Additionally, RASPT2 ${ }^{32,74}$ can be added to compute the correlation energy. The cost of the integrals does not scale with the number of states, but both RASPT2 and RASSI do. For $\mathrm{MnO}_{2}^{+}$for example, the RASPT2 calculation for all the states took about four and a half weeks of computer hours, while RASSI took less than a day. For this calculation, RASSCF went from more than five weeks with Version 2 to three days with Version 4. This shows that even though RASPT2 is a significant part of the total computation time, the improvements described here offer large time savings in addition to increased stability. This is even more true as the RASPT2 calculation can easily be split across different computer nodes as long as a multi-state calculation is not required 
while the RASSCF code is not as easily parallelisable.

\section{CONCLUSIONS}

RAS wavefunctions offer a flexible route to select important configurations. With the projection technique described here, states with both single and double core holes can be generated directly from the Davidson algorithm. This makes it possible to calculate a wide range of X-ray spectroscopies, including X-ray absorption as well as processes involving two different core orbitals, like non-resonant emission and resonant inelastic X-ray scattering.

Most X-ray processes require a large number of final states to be calculated, which leads to numerical problems in standard Davidson algorithms designed for only a few low-lying excited states. For calculations of transition-metal complexes with hundreds of states the solver often diverges due to linear dependency among the many correction vectors. Simply reducing the number of vectors makes convergence more stable but also slower. Significant reductions in computational time can be reached by adding additional orthonormalization steps to prevent linear dependency, to set a cap for the number of kept correction vectors, and by not calculating correction vectors for states that have already converged. With all changes implemented, the new algorithm outperforms all other versions in terms of time, and in most cases also in terms of the number of iterations. As expected, the best timing improvements are for cases where the CI step dominates the total computational time, that is large active spaces and/or small molecules. For systems with valence spaces of ten active orbitals, the savings in computational time are between 3 and 15, with the latter value for the smallest system. In the end, as no case was found where Version 4 performs worse, it has been set as the default option in OpenMolcas.

The combination of a flexible MCSCF wavefunction, an efficient CI algorithm, and possibilities to calculate transition intensities using several different representations of the electromagnetic wavevector now makes OpenMolcas a powerful tool to simulate a wide range of $\mathrm{X}$-ray spectroscopic techniques, even for systems that require large active spaces. 


\section{ACKNOWLEDGMENTS}

We acknowledge financial support from the foundation Olle Engkvist Byggmästare (M.G.D.), the Carl Trygger foundation (R.C.C.) and the Knut and Alice Wallenberg Foundation (Grant No. KAW-2013.0020). 


\section{References}

1. E. C. Wasinger, F. M. De Groot, B. Hedman, K. O. Hodgson, and E. I. Solomon, J. Am. Chem. Soc 125, 12894 (2003).

2. P. Norman and A. Dreuw, Chemical Reviews 118, 7208 (2018).

3. A. Barth and J. Schirmer, Journal of Physics B: Atomic and Molecular Physics 18, 867 (1985).

4. T. Fransson, D. R. Rehn, A. Dreuw, and P. Norman, The Journal of Chemical Physics 146, 094301 (2017).

5. M. Nooijen and R. J. Bartlett, The Journal of Chemical Physics 102, 6735 (1995).

6. S. Coriani, O. Christiansen, T. Fransson, and P. Norman, Phys. Rev. A 85, 022507 (2012).

7. S. Coriani and H. Koch, The Journal of Chemical Physics 143, 181103 (2015).

8. T. Akama and H. Nakai, The Journal of Chemical Physics 132, 054104 (2010).

9. N. A. Besley and F. A. Asmuruf, Phys. Chem. Chem. Phys. 12, 12024 (2010).

10. W. Butscher, R. J. Buenker, and S. D. Peyerimhoff, Chemical Physics Letters 52, 449 (1977), ISSN 0009-2614.

11. H. J. A. Jensen, P. Jørgensen, and H. Ågren, The Journal of chemical physics 87, 451 (1987).

12. U. Ekström, P. Norman, V. Carravetta, and H. Ågren, Phys. Rev. Lett. 97, 143001 (2006).

13. W. Liang, S. A. Fischer, M. J. Frisch, and X. Li, Journal of Chemical Theory and Computation 7, 3540 (2011).

14. D. Zuev, E. Vecharynski, C. Yang, N. Orms, and A. I. Krylov, Journal of Computational Chemistry 36, 273 (2014). 
15. L. S. Cederbaum, W. Domcke, and J. Schirmer, Phys. Rev. A 22, 206 (1980).

16. A. Sadybekov and A. I. Krylov, The Journal of Chemical Physics 147, 014107 (2017).

17. T.-C. Jagau, K. B. Bravaya, and A. I. Krylov, Annual Review of Physical Chemistry 68, 525 (2017), pMID: 28463649.

18. J. Liu, D. Matthews, S. Coriani, and L. Cheng, Journal of Chemical Theory and Computation 0, null (2019), pMID: 30702889.

19. H. Ågren and H. J. Aa. Jensen, Chemical physics letters 137, 431 (1987).

20. R. Klooster, R. Broer, and M. Filatov, Chemical Physics 395, 122 (2012).

21. I. Josefsson, K. Kunnus, S. Schreck, A. Föhlisch, F. de Groot, P. Wernet, and M. Odelius, J. Phys. Chem. Lett. 3, 3565 (2012).

22. P. Wernet, K. Kunnus, S. Schreck, W. Quevedo, R. Kurian, S. Techert, F. M. de Groot, M. Odelius, and A. Föhlisch, J. Phys. Chem. Lett. 3, 3448 (2012).

23. S. I. Bokarev, M. Dantz, E. Suljoti, O. Kühn, and E. F. Aziz, Phys. Rev. Lett. 111, 083002 (2013).

24. N. Engel, S. I. Bokarev, E. Suljoti, R. Garcia-Diez, K. M. Lange, K. Atak, R. Golnak, A. Kothe, M. Dantz, O. Kühn, et al., J. Phys. Chem. B. 118, 1555 (2014).

25. R. V. Pinjari, M. G. Delcey, M. Guo, M. Odelius, and M. Lundberg, J. Chem. Phys 141, $124116(2014)$.

26. R. V. Pinjari, M. G. Delcey, M. Guo, M. Odelius, and M. Lundberg, J. Chem. Phys 142, 069901 (2015).

27. P. Wernet, K. Kunnus, I. Josefsson, I. Rajkovic, W. Quevedo, M. Beye, S. Schreck, S. Gruebel, M. Scholz, D. Nordlund, et al., Nature. 520, 78 (2015).

28. K. Kunnus, W. Zhang, M. G. Delcey, R. V. Pinjari, P. S. Miedema, S. Schreck, W. Quevedo, H. Schroeder, A. Föhlisch, K. J. Gaffney, et al., J. Phys. Chem. B. 120, $7182(2016)$. 
29. A. Chantzis, J. K. Kowalska, D. Maganas, S. DeBeer, and F. Neese, Journal of Chemical Theory and Computation 14, 3686 (2018).

30. J. Olsen, B. O. Roos, P. Jørgensen, and H. J. A. Jensen, J. Chem. Phys 89, 2185 (1988).

31. P. A. Malmqvist, A. Rendell, and B. O. Roos, J. Phys. Chem 94, 5477 (1990).

32. P.-Å. Malmqvist, K. Pierloot, A. R. M. Shahi, C. J. Cramer, and L. Gagliardi, J. Chem. Phys 128, 204109 (2008).

33. P.-Å. Malmqvist and B. O. Roos, Chem. Phys. Lett. 155, 189 (1989).

34. P.-Å. Malmqvist, B. O. Roos, and B. Schimmelpfennig, Chem. Phys. Lett. 357, 230 (2002).

35. S. Bernadotte, A. J. Atkins, and C. R. Jacob, The Journal of Chemical Physics 137, 204106 (2012).

36. M. Guo, L. K. Sørensen, M. G. Delcey, R. V. Pinjari, and M. Lundberg, Phys. Chem. Chem. Phys 18, 3250 (2016).

37. L. K. Sørensen, M. Guo, R. Lindh, and M. Lundberg, Mol. Phys 115, 174 (2017).

38. N. H. List, J. Kauczor, T. Saue, H. J. A. Jensen, and P. Norman, The Journal of chemical physics 142, 244111 (2015).

39. N. H. List, T. Saue, and P. Norman, Molecular Physics 115, 63 (2017).

40. L. K. Sørensen, E. Kieri, S. Srivastav, M. Lundberg, and R. Lindh, Physical Review A (In press).

41. E. Suljoti, R. Garcia-Diez, S. I. Bokarev, K. M. Lange, R. Schoch, B. Dierker, M. Dantz, K. Yamamoto, N. Engel, K. Atak, et al., Angewandte Chemie International Edition 52, 9841 (2013).

42. M. Guo, E. Källman, R. V. Pinjari, , R. Couto Carvalho, L. K. Sørensen, R. Lindh, K. Pierloot, and M. Lundberg, Journal of Chemical Theory and Computation (2018). 
43. F. De Groot, Journal of Electron Spectroscopy and Related Phenomena 67, 529 (1994).

44. B. Gilbert, B. Frazer, A. Belz, P. Conrad, K. Nealson, D. Haskel, J. Lang, G. Srajer, and G. De Stasio, The Journal of Physical Chemistry A 107, 2839 (2003).

45. M. Kubin, M. Guo, T. Kroll, H. Löchel, E. Källman, M. L. Baker, R. Mitzner, S. Gul, J. Kern, A. Föhlisch, et al., Chem. Sci. (2018).

46. R. V. Pinjari, M. G. Delcey, M. Guo, M. Odelius, and M. Lundberg, J. Comput. Chem 37, 477 (2016).

47. R. K. Hocking, E. C. Wasinger, F. M. de Groot, K. O. Hodgson, B. Hedman, and E. I. Solomon, J. Am. Chem. Soc 128, 10442 (2006).

48. "https://gitlab.com/Molcas/OpenMolcas".

49. E. R. Davidson, J. Comput. Phys. 17, 87 (1975).

50. J. Olsen, P. Jørgensen, and J. Simons, Chemical Physics Letters 169, 463 (1990), ISSN 0009-2614.

51. T. Helgaker, P. Jørgensen, and J. Olsen, Configuration-Interaction Theory (John Wiley \& Sons, Ltd, 2014), chap. 11, pp. 523-597, ISBN 9781119019572.

52. M. Guo, E. Källman, L. K. Sørensen, M. G. Delcey, R. V. Pinjari, and M. Lundberg, J. Phys. Chem. A 120, 5848 (2016).

53. S. I. Bokarev, M. Khan, M. K. Abdel-Latif, J. Xiao, R. Hilal, S. G. Aziz, E. F. Aziz, and O. Kuhn, The Journal of Physical Chemistry C 119, 19192 (2015).

54. R. C. Couto, M. Guarise, A. Nicolaou, N. Jaouen, G. S. Chiuzbăian, J. Lüning, V. Ekholm, J.-E. Rubensson, C. Såthe, F. Hennies, et al., Phys. Rev. A 93, 032510 (2016).

55. T. Fleig, J. Olsen, and C. M. Marian, The Journal of Chemical Physics 114, 4775 (2001).

56. P.-Å. Malmqvist, International Journal of Quantum Chemistry pp. 479-494 (1986). 
57. D. Zuev, K. B. Bravaya, T. D. Crawford, R. Lindh, and A. I. Krylov, The Journal of Chemical Physics 134, 034310 (2011).

58. M. Douglas and N. M. Kroll, Ann. Phys. 82, 89 (1974).

59. B. A. Hess, Phys. Rev. A 33, 3742 (1986).

60. B. O. Roos, R. Lindh, P.-A. Malmqvist, V. Veryazov, and P.-O. Widmark, J. Phys. Chem. A. 108, 2851 (2004).

61. B. O. Roos, R. Lindh, P.-Å. Malmqvist, V. Veryazov, and P.-O. Widmark, J. Phys. Chem. A. 109, 6575 (2005).

62. F. Aquilante, T. B. Pedersen, R. Lindh, B. O. Roos, A. Snchez de Mers, and H. Koch, The Journal of Chemical Physics 129, 024113 (2008).

63. L. S. Cederbaum, F. Tarantelli, A. Sgamellotti, and J. Schirmer, The Journal of Chemical Physics 85, 6513 (1986).

64. H. Ågren and H. J. A. Jensen, Chemical physics 172, 45 (1993).

65. J. H. D. Eland, M. Tashiro, P. Linusson, M. Ehara, K. Ueda, and R. Feifel, Phys. Rev. Lett. 105, 213005 (2010).

66. M. Nakano, F. Penent, M. Tashiro, T. P. Grozdanov, M. Žitnik, S. Carniato, P. Selles, L. Andric, P. Lablanquie, J. Palaudoux, et al., Phys. Rev. Lett. 110, 163001 (2013).

67. S. Carniato, P. Selles, P. Lablanquie, J. Palaudoux, L. Andric, M. Nakano, Y. Hikosaka, K. Ito, T. Marchenko, O. Travnikova, et al., Phys. Rev. A 94, 013416 (2016).

68. R. Feifel, J. H. D. Eland, S. Carniato, P. Selles, R. Püttner, D. Koulentianos, T. Marchenko, L. Journel, R. Guillemin, G. Goldsztejn, et al., Scientific reports 7, $13317 ; 13317$ (2017).

69. M. Tronc, G. C. King, and F. H. Read, Journal of Physics B: Atomic and Molecular Physics 12, 137 (1979). 
70. G. Wight and C. Brion, Journal of Electron Spectroscopy and Related Phenomena 4, 335 (1974), ISSN 0368-2048.

71. S. A. Wilson, T. Kroll, R. A. Decreau, R. K. Hocking, M. Lundberg, B. Hedman, K. O. Hodgson, and E. I. Solomon, J. Am. Chem. Soc 135, 1124 (2013).

72. K. Pierloot, Mol. Phys 101, 2083 (2003).

73. F. Aquilante, P.-A. Malmqvist, T. B. Pedersen, A. Ghosh, and B. O. Roos, J. Chem. Theory. Comput. 4, 694 (2008).

74. K. Andersson, P.-A. Malmqvist, B. O. Roos, A. J. Sadlej, and K. Wolinski, Journal of Physical Chemistry 94, 5483 (1990). 